\title{
Special Issue on "advanced distributed wireless communication techniques - theory and practice"
}

\author{
Jan Mietzner ${ }^{1 *}$, Dirk Wübben ${ }^{2}$, Diomidis Michalopoulos ${ }^{3}$, Robert Schober ${ }^{3}$, Peter M Rost ${ }^{4}$ \\ and Christoph Mecklenbräuker ${ }^{5}$
}

\section{Editorial}

In order to keep up with the continuously growing demand for high-rate wireless services, the last decade has witnessed extensive efforts and innovative solutions for enhancing the capacity and reliability of wireless communication systems. Concerning the physical layer, multiple-antenna techniques were shown to offer both diversity and multiplexing gains and were thus readily adopted by standardization bodies and by the wireless industry. In addition, relaying techniques bear the potential of increasing the coverage area of wireless networks and alleviating the impact of link failures. In distributed wireless communication systems these key techniques have been merged, as multiple antennas are no longer colocated, but distributed over several network nodes. By this means, even single-antenna nodes with small form factors can potentially enjoy the benefits of multiple antennas - in addition to the benefits of conventional relaying. Recently, novel ideas from wired networks have started to blend into these concepts in the form of physical-layer network coding schemes. Here, encoding functions are realized by cooperating wireless nodes rather than by a single encoder, as traditionally employed in point-to-point links. By this means, the reliability and throughput of wireless networks can be further improved.

This Special Issue aims to capture the current state of the art of advanced distributed wireless communication techniques, such as distributed space-time coding and distributed beamforming (DBF) schemes, coordinated multipoint (CoMP) and multiuser techniques, multipleinput multiple-output (MIMO) relaying, network coding, and cognitive radio-related techniques. Special focus is on physical-layer aspects as well as on medium-access (MAC) layer design issues for the above techniques, analytical investigations concerning performance prediction and fundamental limits, aspects of non-perfect channel

\footnotetext{
* Correspondence: jan.mietzner@cassidian.com

${ }^{1}$ Cassidian (EADS), Ulm, Germany

Full list of author information is available at the end of the article
}

knowledge and non-perfect feedback information, and implementation aspects in the context of existing wireless standards, such as long-term evolution (LTE) or wireless fidelity (WiFi).

In response to our Call-for-Papers, we received in total 29 paper submissions from researchers around the globe, which underline the high interest in this area. After a rigorous peer-review process, which involved three or more independent expert reviewers and at least two review rounds, we were finally able to accept 16 articles for publication in this Special Issue (three of the rejected papers were outside scope and were thus rejected directly after initial submission). This corresponds to an overall acceptance rate of $55.2 \%$, which reflects the high quality of the accepted contributions.

Within the scope outlined above, the 16 featured articles cover a relatively wide spectrum of timely topics:

- One article addresses fundamental limits of MIMO relaying.

- Four articles fall into the broad area of design and performance analysis of special relaying and distributed precoding schemes.

- Two articles focus on detection algorithms for relayassisted wireless systems.

- Four articles investigate new algorithms and protocols for relay-assisted multiuser systems and cognitive radio networks.

- Five articles are concerned with the applicability of advanced distributed wireless techniques in the context of LTE-advanced (LTE-A) and WiFi systems.

Figure 1 illustrates the spectrum of covered topics. Below, the contents of the individual articles are briefly summarized.

\section{Fundamental limits of relaying schemes}

The paper "Bounds on the capacity regions of halfduplex Gaussian MIMO relay channels" by L. Gerdes, 


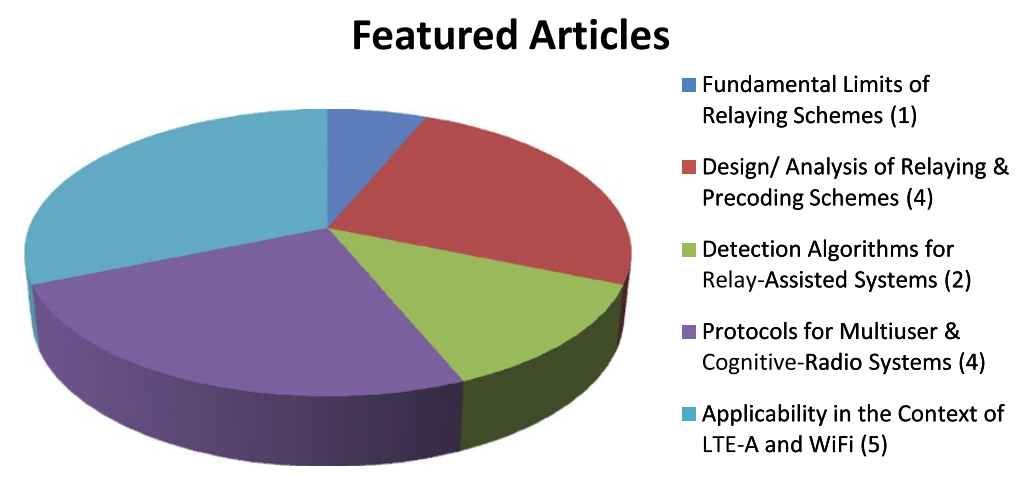

Figure 1 Overview of topics covered by the Special Issue.

M. Riemensberger, and W. Utschick analyzes the halfduplex Gaussian MIMO relay channel and considers both unidirectional and bidirectional communication. The authors derive inner and outer bounds for the capacity region based on a dual decomposition approach, which separates the joint optimization of selecting the channel input signal and the resource assignment in a half-duplex channel. The actual solution to the formulated problem is found through a convex optimization. Furthermore, the authors describe the inherent flexibility of their approach and discuss numerical results for selected scenarios.

\section{Design and performance analysis of relaying and distributed precoding schemes}

In the paper "Wireless MIMO switching: distributed zeroforcing and MMSE relaying using network coding" by M. Wang, F. Wang, and Z. Zhong, DBF design for a group of single-antenna relays is considered. This group of relays acts as a wireless MIMO switch supporting the bidirectional data exchange between several user pairs. The authors propose a distributed zero-forcing beamformer and investigate the required number of relays. In order to improve the throughput, a minimum-mean-squared error(MMSE) based scheme is derived, which requires a larger overhead. Both schemes provide improved system throughput compared to existing distributed schemes.

In their paper "Performance of distributed beamforming for dense relay deployments in the presence of limited feedback information", T. Halinen, A.A. Dowhuszko, and J. Hämäläinen investigate the performance of a DBF algorithm for different amounts of channel phase feedback information. The analysis refers to a particular wireless communication scheme where the main transmitter (subscriber terminal) communicates with a main receiver (base station) via a cluster of cooperative network elements. The resulting cooperative network consists of a large number of array elements which are located in the proximity of the subscriber. The performance measures considered are the outage probability, the bit error rate, and the ergodic capacity, yielding closed-form expressions which offer useful off-line assessment of the deployed scheme. The resulting analysis shows that considerable performance gains can be achieved for a small amount of phase feedback information, such that the overall performance approximates the ideal case with full phase feedback information.

The paper "A distributed approach to precoder selection using factor graphs for wireless communication networks" by I.M. Guerreiro, D. Hui, and C.C. Cavalcante introduces a close-to-optimum message-passing algorithm to select precoders in a multi-cell scenario. The authors consider two particular applications: transmitantenna selection and beam selection. The introduced algorithm is based on a factor graph reflecting the exchange of parameters between individual nodes. The algorithm is compared with an approach where each node exploits the parameters chosen by its immediate neighborhood in the past. Both algorithms are compared in terms of sum-rate performance as well as complexity.

In the paper "Linear precoding in distributed MIMO systems with partial CSIT" by C. Xu, J. Zhang, F. Zheng, and Li Ping, a distributed MIMO scheme with multiple distributed transmitters and a single common receiver is studied. The authors propose a linear precoding strategy which is decentralized and simple to implement, rendering it suitable for distributed MIMO setups. The proposed precoder consists of two parallel modules, which perform channel matching and energy scattering, respectively, offering thus a coherent gain with improved power efficiency as well as robustness against imperfect transmitside channel state information (CSIT). Theoretical conclusions are corroborated by numerical examples.

\section{Detection algorithms for relay-assisted wireless systems}

The article "Outage probability of a relay strategy allowing intra-link errors utilizing Slepian-Wolf theorem" by $M$. Cheng, K. Anwar, and T. Matsumoto considers a relaying strategy that is based on the Slepian-Wolf (SW) theorem originally formulated for the compression of correlated sources. As opposed to conventional decode-and-forward 
(DF) relaying, where erroneous data blocks at the relay are discarded, the proposed SW relaying scheme allows for intra-link errors between source and relay, while employing a joint decoding scheme at the destination which exploits the correlation between the source signal and the relayed signal. The authors provide novel integral expressions for the resulting outage probability as well as asymptotic results illustrating the impact of intra-link errors on the resulting diversity order. The theoretical results are corroborated by simulation results obtained for a SW relaying scheme based on bitinterleaved coded modulation with iterative detection.

In their paper "Reliability-aware iterative detection scheme (RAID) for distributed IDM space-time codes in relay systems", F. Lenkeit, D. Wübben, and A. Dekorsy propose an iterative reliability-aware detection scheme for multi-user two-hop DF relaying scenarios which is based on distributed interleave-division multiplexing (IDM) space-time codes. The main goal of the proposed scheme is to combat error propagation by the DF relay, which occurs whenever the relay terminal erroneously detects the received signal. This is achieved by distinguishing between the detection of the relay messages and the subsequent estimation of the source messages, such that signals from erroneous relays are processed separately from the signals incident from successful relays. The proposed scheme is shown to offer considerable performance improvements to conventional detection. Moreover, the proposed scheme is shown to outperform adaptive relaying in certain scenarios.

\section{Algorithms and protocols for relay-assisted multiuser systems and cognitive radio networks}

In their paper "Resource allocation for the multiple-access relay channels and OFDMA", S. Schedler and V. Kühn propose resource allocation algorithms for relay-aided orthogonal frequency-division multiple access (OFDMA) schemes. In particular, a centralized optimization framework is introduced aiming at maximizing the network's achievable sum rate across all subcarriers. The proposed approach is applied to relay setups such as amplify-and-forward, DF (both adaptive and non-adaptive), as well as compress-and-forward. The resulting optimization problem involving individual transmit power constraints is tackled using two approaches: (a) by dividing the overall problem into the problems of individual power allocation and carrier allocation and (b) by adopting relaxation of the constraints. Using an extensive set of simulations, the complexity of the proposed algorithms as well as their performance in terms of achieved sum rates are assessed. It is concluded that the performance of the proposed algorithms approach the optimal performance.

In their paper "A distributed algorithm using interference pricing for relay interference channels", K.T. Truong and R.W. Heath tackle the issue of interference in relaybased cellular systems, where a set of relays forwards the information from a set of transmitters to a set of receivers. A two-step algorithm for the design of the precoders at the transmitters and the relays is presented. The first step of the proposed algorithm deals with existing single-hop approaches for designing the precoders at the transmitters. The second step capitalizes on the concept of interference pricing, and it develops a distributed algorithm, in which each relay forms its own transmit precoder without knowledge of the precoders of the other relays. The proposed two-hop interference pricing method uses an approximation function for the end-to-end achievable rate, thereby taking into account the relation of the channel strength on the links between the transmitters and the relays and from relays to the receivers. It is shown that the proposed algorithm outperforms relevant algorithms in terms of the endto-end sum rate, for a variety of system configurations.

In their article "Weighted sum-rate maximization for multi-user SIMO multiple access channels in cognitive radio networks", P. He, L. Zhao, and J. Lu consider a concurrent spectrum access scenario between two separate groups of users served by a common base station. The primary user (PU) group consists of licensed users of the frequency band under consideration, while the secondary user (SU) group is allowed to utilize the same band under the constraint that the interference level to the PUs caused by the SUs does not exceed a certain limit. With focus on the uplink and the single-input multiple-output (SIMO) case, the authors formulate a corresponding weighted sum-rate maximization problem for the information rates of the SUs and devise a novel iterative waterfilling algorithm to find the optimum solution. As opposed to existing algorithms, the proposed iterative waterfilling algorithm has the desired property of delivering a sequence of feasible solutions across iterations. Moreover, by exploiting the particular problem structure, the optimization is decoupled for the individual SUs which allows for efficient parallel processing and fast convergence, even for a large number of SUs.

The paper "A concurrent access MAC protocol for cognitive radio ad hoc networks without common control channel" by S.K. Timalsina, S. Moh, I. Chung, and M. Kang considers a typical problem in a cognitive radio ad hoc network: spatially distributed SUs do not necessarily have the same channels available for dynamic spectrum access, because they may observe different activity of PUs. Correspondingly, it cannot be guaranteed that there is at least one channel common to all SUs, which makes it difficult to install a common control channel (CCC) for secondary medium access. To this end, the authors propose a concurrent access MAC (CA-MAC) protocol, which does not require any $\mathrm{CCC}$ and limits the waiting time for the secondary nodes by allowing parallel channel access by 
multiple communication pairs within the same time slot. Analytical and simulation results illustrate the superiority of the proposed CA-MAC protocol with regard to the resulting throughput when compared to state-of-the-art MAC protocols without CCC.

\section{Applicability of advanced distributed wireless techniques in LTE-advanced and WiFi systems}

In their paper "Interference coordination of heterogeneous LTE systems using remote radio heads", J. Kim, D. Lee, and W. Sung consider the problem of co-channel interference (CCI) mitigation in LTE-advanced heterogeneous networks (HetNets). In contrast to a homogeneous cell structure, which is based on a single type of serving nodes - the macro base stations (BSs), HetNets employ multiple types of serving nodes for coverage extension. In this paper, focus is on HetNets composed of macro BSs and remote radio heads (RRHs) - smaller serving nodes which are geographically distributed and connected to the macro BSs via fast and reliable backhaul links. The authors first discuss two available CCI mitigation techniques for LTE-advanced HetNets and then propose a novel CCI mitigation scheme which uses temporary muting of RRHs. System-level simulations illustrate the advantages of the proposed scheme in terms of achieved signal-to-noise-and-interference ratios and user throughput. Deployment options with regard to geographical placement of RRHs are also investigated.

The paper "System-level performance of LTE-advanced with joint transmission and dynamic point selection schemes" by H.-L. Määttänen, K. Hämäläinen, J. Venäläinen, K. Schober, M. Enescu, and M. Valkama analyzes different CoMP algorithms which are standardized in the context of 3GPP LTE such as interference coordination, joint transmission, and dynamic point selection. The authors consider practical aspects such as finite-rate feedback used to determine the mode of cooperation and as input for the cooperation algorithm itself. Using an extensive system-level simulation campaign, which also considers non-cooperative transmission as a fallback option, the authors first detail the scenarios and deployments, where gains can be expected, and then quantify the expected gains.

In the paper "Single-user MIMO versus multi-user MIMO in distributed antenna systems with limited feedback" by S. Schwarz, R.W. Heath, and M. Rupp, distributed antenna systems are analyzed where multiple remote radio units and a managing macro BS jointly transmit and receive. The authors discuss the throughput performance of single-user and multi-user MIMO transmission for this setup and show, in which scenarios individual strategies are preferable. A particular emphasis is paid on imperfect channel state information (CSI) and quantization of CSI. The authors discuss how the choice of codebooks can be optimized. Finally, the paper compares the distributed antenna system against a MIMO system with co-located antennas based on a 3GPP LTE compliant system-level simulator.

The paper "A novel type II relay-assisted retransmission scheme for uplink of LTE-advanced system" by A. Li, S. Nagata, A. Harada, and H. Suda considers the applicability of incremental relaying to 3GPP LTE. In this setup, a relay observes the communication between a mobile terminal and a BS. If, in the uplink, the BS is unable to decode correctly, the relay will provide a copy of the signal in order to gain from cooperative diversity. In the case of a type II relay (transparent relay), it is difficult to implement this approach. The authors discuss the constraints and pay particular attention to the control signaling and pilot structure. They propose a novel relaying method which overcomes these restrictions and allows for leveraging the cooperative diversity in a 3GPP LTE relaying scenario.

In their paper "Coded unicast downstream traffic in a wireless network: analysis and WiFi implementation", A. Cohen, E. Biton, J. Kampeas, and O. Gurewitz present a feedback-based coding scheme for multiple unicast communications from a single access point (AP) to multiple users. In this scheme, the users deliver acknowledgements for successfully received packets intended for them as well as for other users. Based on this feedback, the AP combines several packets by network coding to be broadcasted in the next slot. By introducing a state model denoting correctly received overheard packets not been successfully decoded by the intended user, a Markov decision process is derived. Based on this approach, a greedy and a semigreedy algorithm to determine transmission policies are derived. After analyzing the two users' case in detail, the authors present a practical WiFi implementation with off the shelf devices and demonstrate the achieved performance improvements.

We hope that the featured articles will be of interest for the readers of the EURASIP Journal of Advances in Signal Processing (JASP) and that they will foster further research in this exciting area of wireless communications.

\section{Competing interests}

The authors declare that they have no competing interests.

\section{Authors' information}

JM was born in Rendsburg, Germany in 1975. He studied electrical engineering at the Christian-Albrechts University (CAU) of Kiel, Germany, with focus on digital communications. During his studies, he spent 6 months in 2000 with the Global Wireless Systems Research Group, Lucent Technologies, Bell Labs UK, in Swindon, England. He received the Dipl.-Ing. degree from the CAU Kiel in July 2001. From August 2001 to October 2006, he was working toward his Ph.D. degree as a research and teaching assistant at the Information and Coding Theory Lab, CAU Kiel, and received his Ph.D. degree in December 2006. He received an award from the Friends of the Faculty of Engineering for the best dissertation in 2006. From January 2007 to

December 2008, he was with the Department of Electrical and Computer Engineering at the University of British Columbia, in Vancouver, Canada, as a post-doctoral research fellow sponsored by the German Academic Exchange Service (DAAD). In March 2009, he joined Cassidian (EADS), in Ulm, Germany. His research interests concern physical layer aspects of future wireless 
communication systems, especially multiple-antenna techniques, ultrawideband and cognitive radio systems, relaying, and cooperative diversity techniques, as well as theoretical aspects of jamming and radar systems. He has published 40 papers in international journals and conference proceedings and is a co-recipient of the 2010 Best Paper Award from the German Information Technology Society (VDE/ITG). JM has also served as a TPC member for several international conferences such as IEEE Globecom and ICC, and he is a Senior Member of the IEEE.

DW received the Dipl.-Ing. $(\mathrm{FH})$ degree in electrical engineering from the University of Applied Science Münster, Germany, in 1998, and the Dipl.-Ing. (Uni) degree and the Dr.-Ing. degree in electrical engineering from the University of Bremen, Germany in 2000 and 2005, respectively. He was a visiting student at the Daimler Benz Research Departments in Palo Alto, California in 1997, and in Stuttgart, Germany in 1998. From 1998 to 1999, he was with the Research and Development Center of Nokia Networks, Düsseldorf, Germany. In 2001, he joined the Department of Communications Engineering, University of Bremen, Germany, where he is currently a senior researcher and lecturer. His research interests include wireless communications, signal processing for multiple antenna systems, cooperative communication systems, and channel coding. DW was a technical program co-chair of the 2010 International ITG Workshop on Smart Antennas and has been a member of the program committee of several international conferences. His doctoral dissertation on reduced complexity detection algorithms for MIMO systems received the Bremer Studienpreis in 2006.

DSM was born in Thessaloniki, Greece in 1983. He received a Diploma and a $\mathrm{PhD}$ degree from the Electrical and Computer Engineering Department of the Aristotle University of Thessaloniki in 2005 and 2009, respectively. Since 2009, he has been with the University of British Columbia, Canada, as a Killam postdoctoral fellow, and since 2011 as a Banting postdoctoral fellow. Currently, he is with the University of Erlangen-Nuremberg, Germany, as visiting researcher. His research spans in the broad area of wireless communications in fading channels, with a particular focus on the analysis and optimization of the physical layer of cooperative systems. DSM received the Marconi Young Scholar award in 2010. He is co-recipient of the Best Paper Award of the Wireless Communications Symposium (WCS) in IEEE International Conference on Communications (ICC '07). He is also associate Editor for the IEEE Communications Letters.

RS was born in Neuendettelsau, Germany in 1971. He received the Diplom (Univ.) and the Ph.D. degrees in electrical engineering from the University of Erlangen-Nuremberg, Germany, in 1997 and 2000, respectively. From May 2001 to April 2002, he was a Postdoctoral Fellow at the University of Toronto, Canada, sponsored by the German Academic Exchange Service (DAAD). Since May 2002, he has been with the University of British Columbia (UBC), Vancouver, Canada, where he became a Full Professor and Canada Research Chair (Tier II) in Wireless Communications. Currently, he is a Full Professor at the University of Erlangen-Nuremberg. His research interests fall into the broad areas of communication theory, wireless communications, and statistical signal processing. RS received the 2002 Heinz Maier-Leibnitz Award of the German Science Foundation (DFG), the 2004 Innovations Award of the Vodafone Foundation for Research in Mobile Communications, the 2006 UBC Killam Research Prize, the 2007 Wilhelm Friedrich Bessel Research Award of the Alexander von Humboldt Foundation, and the 2008 Charles McDowell Award for Excellence in Research from UBC. In addition, he received best paper awards from the German Information Technology Society (ITG), the European Association for Signal, Speech and Image Processing (EURASIP), IEEE ICUWB 2006, the International Zurich Seminar on Broadband Communications, and European Wireless 2000. RS is a Fellow of the IEEE. He was also the Area Editor for Modulation and Signal Design for the IEEE Transactions on Communications. Since January 2012, he has been serving as Editor-in-Chief for the IEEE Transactions on Communications.

PR received his Ph.D. degree from Technische Universität Dresden, Dresden, Germany in 2009 and his M.Sc. degree from University of Stuttgart, Stuttgart, Germany in 2005. From 1999 to 2002, he was with Fraunhofer Institute for Beam and Material Technologies, Dresden, Germany where he developed augmented and virtual reality-based controlling systems. From 2002 to 2005, he was with IBM Deutschland Entwicklung GmbH, Böblingen, Germany, where he contributed to the IBM Tivoli system. In June 2005, he joined the Vodafone Chair of Prof. Gerhard Fettweis at Technische Universität Dresden and focused on different aspects of relaying in the context of mobile communications systems. He was also a leading member of the organizing team of IEEE ICC 2009, prepared multiple successful EU FP6 and FP7 proposals, and was actively contributing to the European research project WINNER since 2005 as task leader. Since April 2010, PR is a member of the Mobile and Wireless Networks group at NEC Laboratories Europe, where he is working as Senior Researcher in business unit projects, 3GPP RAN2 as active delegate, and the EU FP7 projects FLAVIA and iJOIN, which he currently manages as Technical Manager (www.ict-ijoin.eu). Among others, PR is a guest editor at Elsevier Journal on Computer Networks and Journal on Advances in Signal Processing; he served as TPC Chair at IEEE VTC Spring 2013; and he is a member of the IEEE ComSoc GITC. PR published more than 30 scientific publications and he is author of multiple patents and patent applications.

CM was born in Darmstadt, Germany in 1967. He received the Dipl-Ing. degree in Electrical Engineering from Vienna University of Technology in 1992 and the Dr.-Ing. degree from Ruhr-University of Bochum in 1998. His doctoral thesis was awarded with the Gert Massenberg Prize. From 1997 to 2000, he worked for the Mobile Networks Radio department of Siemens AG Austria, where he participated in the European framework of ACTS 90 FRAMES. He was a delegate to the Third Generation Partnership Project (3GPP) and engaged in the standardization of the radio access network for UMTS. Since June 2000, he was a senior researcher at the

Telecommunications Research Center Vienna (ftw.) in the field of mobile communications, key researcher since November 2002, and proxy since July 2003. Between 2006 and 2009, he coordinated the Sixth Framework project "Multiple-Access Space-Time Coding Testbed" (MASCOT) on behalf of ftw. He leads the Special Interest Group on mobile-to-mobile communications within COST Action 2100 Pervasive Mobile and Ambient Wireless Communications. In 2006, he joined the Institute of Communications and Radio Frequency Engineering at Vienna University of Technology as a full professor. Since July 2009, he leads the newly founded Christian Doppler Laboratory for Wireless Technologies for Sustainable Mobility. His current research interests include radio interfaces for future peer-to-peer networks (car-to-car communications, personal area networks, and wireless sensor networks), ultra-wideband radio and MIMO-OFDM-based transceivers (UMTS long-term evolution, WiMAX, and $4 \mathrm{G}$ ). CM is a member of the IEEE, the Antennas and Propagation Society, the Vehicular Technology society, the Signal Processing society, and EURASIP. He is the councilor of the IEEE Student Branch Wien. He is also associate editor of the EURASIP Journal of Applied Signal Processing.

\section{Acknowledgements}

We would like to thank all authors for submitting their works to this Special Issue. Moreover, we thank the reviewers for their valuable time and effort spent to evaluate the submitted manuscripts and to suggest possible improvements. Finally, we would like to thank the editorial team of the EURASIP JASP for their continuous support in handling the review process and in compiling this Special Issue.

\section{Author details}

${ }^{1}$ Cassidian (EADS), Ulm, Germany. ${ }^{2}$ University of Bremen, Bremen, Germany. ${ }^{3}$ The University of British Columbia, Vancouver, Canada. ${ }^{4}$ NEC Laboratories Europe, Heidelberg, Germany. ${ }^{5}$ Vienna University of Technology, Vienna, Austria.

Received: 1 September 2013 Accepted: 5 September 2013 Published: 16 September 2013

doi:10.1186/1687-6180-2013-146

Cite this article as: Mietzner et al: Special Issue on "advanced distributed wireless communication techniques - theory and practice". EURASIP Journal on Advances in Signal Processing 2013 2013:146. 